\title{
ADIÇÃO DE FILTRADO INDUSTRIAL NA PRECIPITAÇÃO DE HIDRATO DE ALUMINA NO PROCESSO BAYER*
}

\author{
Roberto Seno Junior ${ }^{1}$ \\ Rodrigo Aparecido Moreno ${ }^{2}$ \\ Thiago Teixeira Franco ${ }^{3}$ \\ Alexandre Rezende de Freitas ${ }^{4}$ \\ Eduardo Marchioni Escobar ${ }^{5}$
}

\section{Resumo}

A precipitação é uma etapa de extrema importância na produção de alumina por influenciar diretamente em vários indicadores de performance, como produtividade, consumo de vapor, estabilidade da planta e muitos outros. Esse processo tem como força motriz a supersaturação de alumina no licor Bayer, que é a diferença entre a concentração de alumina no licor e a sua concentração de equilíbrio. A concentração cáustica no licor impacta a supersaturação. Foi identificada a oportunidade de adição do filtrado da filtração de hidrato $(\mathrm{H} 2)$ no início da precipitação, aumentando a supersaturação de alumina no licor Bayer. Para a mesma produção de alumina, os resultados obtidos foram o aumento da produtividade, aumento da concentração de sólidos no circuito, além da diminuição de licor circulante na refinaria. Este último, por sua vez, influencia diretamente no consumo de vapor da planta, o que implica na redução do uso de gás natural.

Palavras-chave: Precipitação; Hidrato de alumina; Supersaturação; Produtividade; Consumo de vapor.

\section{INDUSTRIAL FILTRATE ADDITION INTO THE BAYER ALUMINA HYDRATE PRECIPITATION}

\section{Abstract}

Precipitation process is a step of major importance in alumina production, as it has direct impact on several refinery performance indicators, like precipitation yield, vapor consumption, plant stability and others. The alumina supersaturation, that is the difference between the alumina concentration in the liquor and its equilibrium concentration, is a huge driving force in the precipitation process. The liquor caustic concentration interferes in the supersaturation value. The addition of the filtrate of hydrate filtration $(\mathrm{H} 2)$ at the beggining of precipitation process, is a great opportunity to increase the alumina supersaturation. For the same alumina production, the benefits of operating the precipitation with higher alumina supersaturation were elevated yield and solids concentration in the precipitation, besides lower refinery liquor flow. The plant steam consumption (and consequently the natural gas consumption) is directly influenced by the refinery liquor flow.

Keywords: Precipitation; Alumina hydrate; Supersaturation; Yield; Steam consumption.

1 Engenharia Química, Engenheiro Químico, Gerente de Tecnologia Alumina, Tecnologia da Alumina, Votorantim Metais / Cia. Brasileira de Alumínio, Alumínio, São Paulo, Brasil.

2 Química, Bacharel, Consultor de Processos, Tecnologia da Alumina, Votorantim Metais / Cia. Brasileira de Alumínio, Alumínio, São Paulo, Brasil.

3 Engenharia Química, Engenheiro Químico, Engenheiro de Processos, Tecnologia da Alumina, Votorantim Metais / Cia. Brasileira de Alumínio, Alumínio, São Paulo, Brasil.

4 Engenharia química, Engenheiro Químico, Engenheiro de $P \& D$, Tecnologia da Alumina, Votorantim Metais / Cia. Brasileira de Alumínio, Alumínio, São Paulo, Brasil.

5 Química, Mestre, Consultor de Processos, Tecnologia da Alumina, Votorantim Metais / Cia. Brasileira de Alumínio, Alumínio, São Paulo, Brasil.

* Contribuição técnica ao $35^{\circ}$ Seminário de Balanços Energéticos Globais e Utilidades e $29^{\circ}$ Encontro de Produtores e Consumidores de Gases Industriais, 13 a 15 de agosto de 2014, São Paulo, SP, Brasil. 


\section{INTRODUÇÃO}

A Companhia Brasileira de Alumínio (CBA) do grupo Votorantim, localiza-se em Alumínio, $74 \mathrm{~km}$ de São Paulo e é a maior fábrica integrada de alumínio no mundo. Com início das operações em 1955, a CBA possui refinaria de alumina, redução eletrolítica, fundição e transformação plástica, além de três sites de mineração no estado de Minas Gerais. Atualmente, sua produção anual é de 0,475 Mt de alumínio primário.

A Precipitação é uma das últimas etapas do processo Bayer de uma refinaria de alumina. Tal processo pode ser divido em duas partes, popularmente conhecidas como área vermelha e área branca. Resumidamente, na área vermelha ocorre a dissolução em solução cáustica do óxido de alumínio tri-hidratado $\left(\mathrm{Al}_{2} \mathrm{O}_{3} .3 \mathrm{H}_{2} \mathrm{O}\right)$ existente no minério de bauxita e a separação dos resíduos do processo; e na área branca, ocorre a precipitação do óxido hidratado e posteriormente a remoção das moléculas de água da estrutura desse óxido (etapa seguinte ao processo Bayer chamada calcinação), gerando o composto conhecido como alumina $\left(\mathrm{Al}_{2} \mathrm{O}_{3}\right)$.

O processo de Precipitação é uma etapa de considerável importância na produção de alumina, pois tem impacto direto em vários indicadores de desempenho de uma refinaria, como por exemplo, na produtividade, no consumo de vapor, na estabilidade da planta, dentre outros.

\section{PROCESSO}

A bauxita das minerações passa pelo processo de moagem a úmido, para diminuição de sua granulometria e depois a polpa formada segue para baterias de trocadores de calor onde sofre aquecimento. Em seguida, esta polpa de bauxita aquecida é enviada a tanques reatores de baixa pressão onde o principal objetivo é a dissolução da sílica reativa e sua posterior precipitação em forma de DSP (Desilication Product).

O processo subsequente pode ser visto no fluxograma simplificado na figura 1. A polpa resultante segue para reatores pressurizados que recebem soda de ataque, principal insumo do processo Bayer, responsável pela digestão da alumina trihidratada ou gibsita $\left(\mathrm{Al}_{2} \mathrm{O}_{3} .3 \mathrm{H}_{2} \mathrm{O}\right)$ do minério, transformando-a em aluminato de sódio $\left(\mathrm{NaAlO}_{2}\right)$ que permanece na fase aquosa. A suspensão resultante, a uma temperatura de aproximadamente $144^{\circ} \mathrm{C}$ passa por tanques flashes onde sofrem perda de vapor para trocadores de calor (os mesmos que aquecem a soda de ataque) e consequente resfriamento para aproximadamente $102^{\circ} \mathrm{C}$.

* Contribuição técnica ao $35^{\circ}$ Seminário de Balanços Energéticos Globais e Utilidades e $29^{\circ}$ Encontro de Produtores e Consumidores de Gases Industriais, 13 a 15 de agosto de 2014, São Paulo, SP, Brasil. 

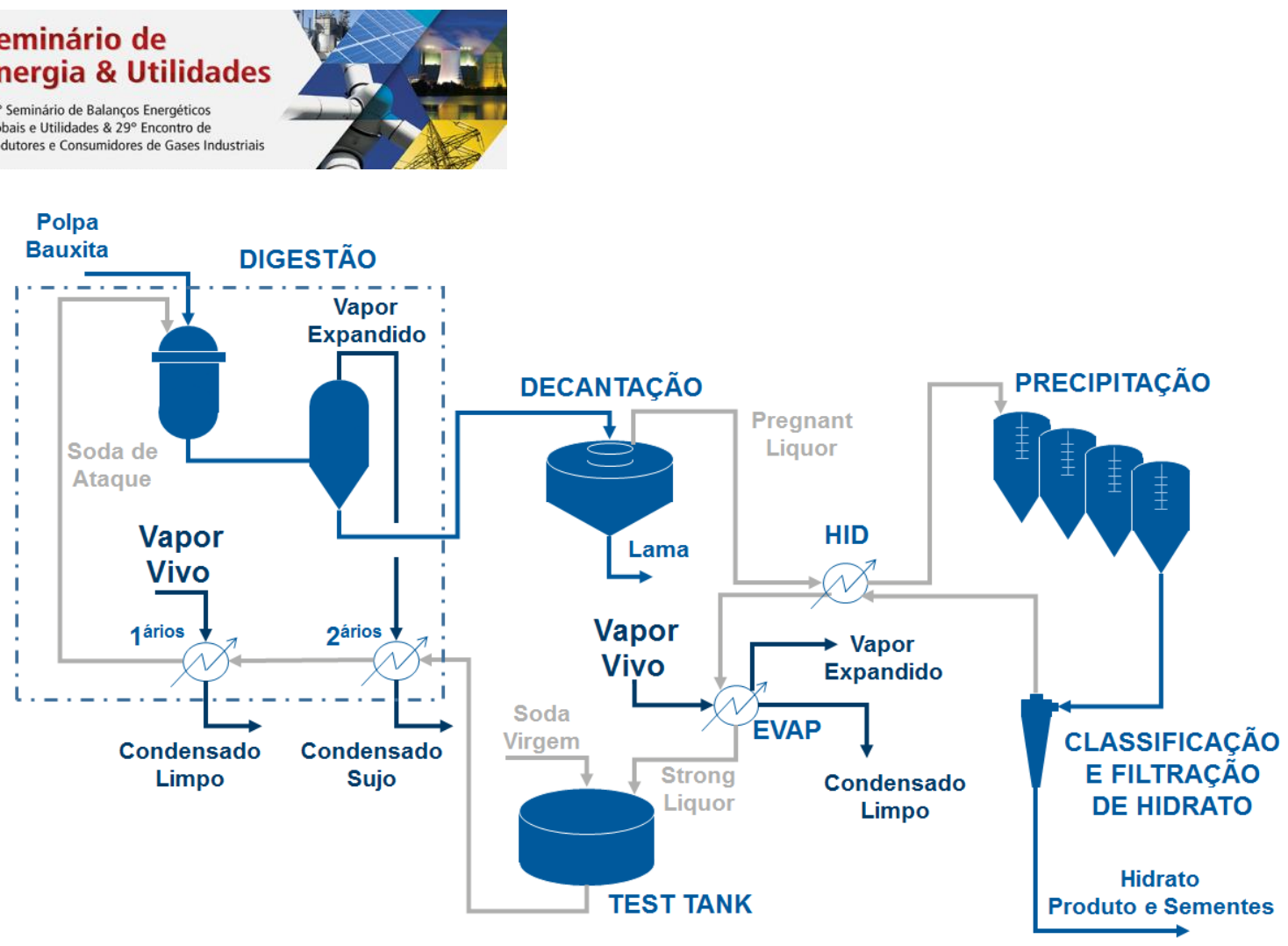

Figura 1. Fluxograma simplificado.

A partir disso, a suspensão segue para um extenso circuito de separação sólidolíquido, com classificadores de areia, decantadores e filtros rotativos, com o objetivo de separar os principais rejeitos do processo como a areia e a lama e recuperar a soda contido nos mesmos.

A parte líquida, separada no overflow do primeiro decantador passa por filtração e é enviada a um sistema de resfriamento, chamado HIDs (High Interchange Departments) de tanques flash e trocadores de calor. Já resfriado, o aluminato de sódio é bombeado aos tanques de precipitação onde a cristalização do hidrato de alumina $\left(\mathrm{Al}_{2} \mathrm{O}_{3} .3 \mathrm{H}_{2} \mathrm{O}\right)$ ocorre. A suspensão resultante segue para um sistema de classificação, onde separa-se o produto final das sementes grossas e finas, as quais retornam ao processo de precipitação. Ambos produtos e sementes passam por etapa de filtração para recuperação do licor de teor cáustico.

Este licor passa pelos trocadores de calor dos HIDs, onde é aquecido. Parte dele segue para tanques de estocagem (test tanks) e parte segue para um sistema de evaporação. Nesse sistema, usa-se vapor vivo para aumentar a temperatura do licor, ocorrendo assim expansão de vapor e concentração do teor cáustico. Este licor concentrado é bombeado aos test tanks.

Após os test tanks, há a correção do teor cáustico do licor, acrescentando-se soda virgem no processo. Essa composição origina a soda de ataque que segue aos trocadores secundários da digestão, os quais aproveitam o vapor expandido dos tanques flash. Logo após, a soda de ataque segue aos trocadores primários da digestão que utilizam vapor vivo para que a temperatura nos reatores pressurizados atinja $144^{\circ} \mathrm{C}$. A soda de ataque segue aos reatores juntamente com a polpa de bauxita, fechando o circuito de licor.

\subsection{Produção de Vapor}

A produção de vapor na refinaria de alumina da CBA ocorre através de 6 caldeiras. Três delas de menor capacidade (35 t/h) queimam óleo e as outras três podem

* Contribuição técnica ao $35^{\circ}$ Seminário de Balanços Energéticos Globais e Utilidades e $29^{\circ}$ Encontro de Produtores e Consumidores de Gases Industriais, 13 a 15 de agosto de 2014, São Paulo, SP, Brasil. 
queimar óleo ou gás natural, com capacidades de produção de 60, 80 e 93 t/h de vapor.

O vapor gerado é consumido nas unidades de digestão e na unidade de evaporação, como ilustrado na Figura 1 e sua quantidade depende do volume circulante de licor no sistema.

\subsection{Precipitação}

A precipitação é o processo de cristalização do hidrato de alumina no meio cáustico, representada pela reação a seguir:

$$
2 \mathrm{NaAlO}_{2}(\mathrm{aq})+4 \mathrm{H}_{2} \mathrm{O}(\mathrm{l}) \rightarrow \mathrm{Al}_{2} \mathrm{O}_{3} \cdot 3 \mathrm{H}_{2} \mathrm{O}(\mathrm{s})+2 \mathrm{NaOH}(\mathrm{aq})
$$

O driving force da precipitação do hidrato é a supersaturação, quimicamente representado pela diferença entre a concentração de alumina no licor (a) e a concentração de equilíbrio de alumina $\left(a^{*}\right)$, a qual é fortemente influenciada pela concentração cáustica do meio.

A cinética da precipitação pode ser expressa pela taxa de crescimento linear $(G)$ dos cristais de hidrato:

$$
\mathrm{G}=\frac{\mathrm{dL}}{\mathrm{dt}}=\mathrm{K}_{\mathrm{g}} \cdot \mathrm{f}(\mathrm{S})
$$

Onde, $\mathrm{L}$ é o tamanho característico dos cristais, $\mathrm{Kg}$ é a constante de crescimento e $\mathrm{S}$ é a supersaturação. A constante de crescimento é influenciada pela temperatura (T) de acordo com a equação de Arrhenius:

$$
\mathrm{K}_{\mathrm{g}}=\mathrm{k} \cdot \mathrm{e}^{\left(-\frac{\mathrm{E}}{\mathrm{RT}}\right)}
$$

Onde, E é energia de ativação da reação de precipitação. Muitos modelos de correlação já foram publicados:

Tabela 1 - Exemplos de modelos cinéticos de precipitação de hidrato [1-4]

\begin{tabular}{lcc}
\hline \multicolumn{1}{c}{ Fonte } & Modelo & E (kJ/mol) \\
\hline White and Misra [1] & $\mathrm{K}_{\mathrm{g}} \cdot\left(a-a^{*}\right)^{2}$ & $60 \pm 6$ \\
King [2] & $\mathrm{K}_{\mathrm{g}} \cdot\left(\frac{a-a^{*}}{F C}\right)^{2}$ & 53 \\
White anda Bateman [3] & $\frac{\mathrm{K}_{\mathrm{g}}}{\sqrt{\mathrm{C}} \cdot\left(\frac{a-a^{*}}{C}\right)^{2}}$ & $71 \pm 7$ \\
Veesler and Boistelle [4] & $\mathrm{K}_{\mathrm{g}} \cdot\left(\frac{a}{a^{*}}-\beta_{c}\right)^{\mathrm{g}}$ & 121 \\
\hline
\end{tabular}

Os modelos propostos e a prática indicam que a supersaturação e o teor cáustico do meio têm relação inversa.

\section{DESENVOLVIMENTO}

A produção de alumina no processo Bayer pode ser calculada através da seguinte fórmula:

* Contribuição técnica ao $35^{\circ}$ Seminário de Balanços Energéticos Globais e Utilidades e $29^{\circ}$ Encontro de Produtores e Consumidores de Gases Industriais, 13 a 15 de agosto de 2014, São Paulo, SP, Brasil. 


\section{RESULTADOS}

O cenário no momento da adição do filtrado, era de produção de $2400 \mathrm{t} / \mathrm{dia}$ de alumina e produtividade de $67,0 \mathrm{~g} / \mathrm{L}$. Ao final, obteve-se ganho de 2,3 g/L de produtividade, ou seja, ela aumentou para $69,3 \mathrm{~g} / \mathrm{L}$.

Para estimar o ganho de projeto com a modificação de processo, mantendo-se a mesma produção e considerando que a produção de vapor é feita inteiramente através de gás natural, alguns fatores foram levados em consideração:
1) $\underset{\text { Consumo Médio }}{\text { Vapor }}=0,112$ t vapor $/ m^{3}$ licor
$\begin{aligned} & \text { 2) } \\ & \text { Eficiência Média } \\ & \text { das Caldeiras }\end{aligned}=14,4 \mathrm{~kg}$ vapor $/ \mathrm{m}^{3}$ gás
3) $\underset{\text { Gás Natural }}{\text { Preço do }}=1,02 R \$ / m^{3} g a ́ s$

\subsection{Cálculo do Impacto Financeiro}

De acordo com a equação de produção de alumina do item 3, calcula-se a vazão de aluminato frio na precipitação, nas duas situações:

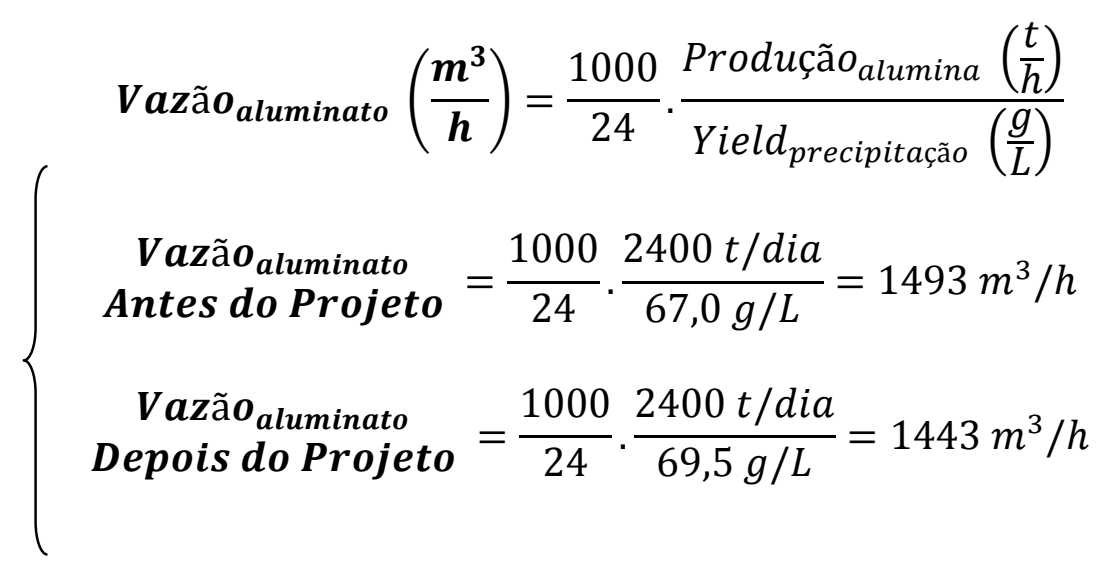

Dessa maneira, a diminuição no fluxo de licor circulante na planta é:

$$
\begin{aligned}
& \text { Diferença de Fluxo } \\
& \text { de Licor }
\end{aligned}=\begin{gathered}
\text { Vazão aluminato } \\
\text { Antes do Projeto }
\end{gathered}-\begin{gathered}
\text { Vazão } o_{\text {aluminato }} \\
\text { Depois do Projeto }
\end{gathered}=1493-1443=50 \mathrm{~m}^{3} / \mathrm{h}
$$

e a diminuição no consumo de vapor:

$\begin{gathered}\text { Diminuição do Consumo } \\ \text { de Vapor }\end{gathered}=\begin{gathered}\text { Diferença de Fluxo Consumo Médio } \\ \text { de Licor }\end{gathered}=50 \frac{\mathrm{m}^{3}}{h} \cdot 0,112 \frac{t}{\mathrm{~m}^{3}}=5,55 \frac{t \text { vapor }}{h}$

Com isso, calcula-se a diminuição do consumo de gás natural nas caldeiras:

$$
\begin{gathered}
\text { Diminuição do Consumo } \\
\text { de Gás nas Caldeiras }
\end{gathered}=\frac{\begin{array}{c}
\text { Diminuição do Consumo } \\
\text { de Vapor }
\end{array}}{\begin{array}{c}
\text { Eficiência Média } \\
\text { das Caldeiras }
\end{array}}
$$

* Contribuição técnica ao $35^{\circ}$ Seminário de Balanços Energéticos Globais e Utilidades e $29^{\circ}$ Encontro de Produtores e Consumidores de Gases Industriais, 13 a 15 de agosto de 2014, São Paulo, SP, Brasil. 


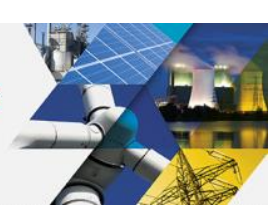

$$
\begin{gathered}
\text { Diminuição do Consumo } \\
\text { de Gás nas Caldeiras }
\end{gathered}=1000 \frac{\mathrm{kg}}{t} \cdot \frac{5,55 \frac{t \text { vapor }}{\mathrm{h}}}{14,4 \frac{\mathrm{kg} \text { vapor }}{\mathrm{m}^{3} \mathrm{gás}}}=385,3 \frac{\mathrm{m}^{3} \mathrm{~g} \text { ás }}{\mathrm{h}}
$$

A partir desse valor é possível calcular a economia no consumo de gás natural e o ganho de projeto anualizado:

$$
\begin{aligned}
& \text { Preço do Diminuição do Consumo } \\
& \begin{array}{c}
\text { Economia no Consumo } \\
\text { de Gás Natural }
\end{array}=\frac{\text { Gás Natural }^{\circ} \text { de Gás nas Caldeiras }}{\text { Produção } o_{\text {alumina }}}
\end{aligned}
$$

$$
\begin{aligned}
& \begin{array}{l}
\text { Economia no Consumo } \\
\text { de Gás Natural }
\end{array}=\frac{1,02 \frac{R \$}{\mathrm{~m}^{3} \text { gás }} \cdot 385,3 \frac{\mathrm{m}^{3} \mathrm{gás}}{\mathrm{h}} \cdot 24 \frac{\mathrm{h}}{\text { dia }}}{2400 \frac{t \text { Alumina }}{\text { dia }}}=3,93 \frac{R \$}{t \text { Alumina }} \\
& \quad \begin{array}{l}
\text { Ganho } \\
\text { Anual }
\end{array}=3,93 \frac{R \$}{t \text { Alumina }} \cdot 2400 \frac{t \text { Alumina }}{\text { dia }} .365 \frac{\text { dia }}{\text { ano }}
\end{aligned}
$$

\section{CONCLUSÃO}

$$
\text { Ganho }=R \$ 3.098 .290,37 / \text { ano }
$$

A precipitação é um processo de mecanismos complexos e a partir do conhecimento de sua teoria, estratégias de controle e melhoria de processo torna-se oportunidades para a diminuição do cash cost do produto final, no caso a alumina.

Com simples manobras de tubulações, foi possível aumentar a supersaturação na cadeia de precipitação através da adição de um filtrado industrial no início desse processo. Por conta disso, a produtividade da precipitação aumentou 2,3 $\mathrm{g} / \mathrm{L}$ e foi possível diminuir o fluxo de licor da planta em $50 \mathrm{~m}^{3} / \mathrm{h}$, mantendo-se a mesma produção de 2400 t/dia de alumina.

Consequentemente, o consumo de gás natural para produção de vapor foi reduzido, o que correspondeu a um ganho anual de aproximadamente $R \$ 3$ milhões.

\section{REFERÊNCIAS}

1 Misra C, White ET. Kinetics of Crystallization of Aluminium Trihydroxide from Seeded Caustic Aluminate Solutions. Chemical Engineering Progress Series 67. 1970;110:5365.

2 King WR. Some Studies in Alumina Trihydrate Precipitation Kinetics. Light Metals. 1973;2:551.

3 White ET, Bateman SH. Effect of Concentration on the Growth Rate of $\mathrm{Al}(\mathrm{OH}) 3$ Particles. Light Metals; 1988;vol:157-162.

4 Veesler S, Boistelle R. About Supersaturation and Growth Rates of Hydragillite $\mathrm{Al}(\mathrm{OH}) 3$ in Alumina Caustic Solutions. Journal of Crystal Growth. 1993;130:411-415.

5 Kenwalt SysCAD. Software de simulação de processos.

* Contribuição técnica ao $35^{\circ}$ Seminário de Balanços Energéticos Globais e Utilidades e $29^{\circ}$ Encontro de Produtores e Consumidores de Gases Industriais, 13 a 15 de agosto de 2014, São Paulo, SP, Brasil. 